\title{
Challenges faced by the curative healthcare system in Sri Lanka
}

\author{
Journal of the Ceylon College of Physicians, 2019, 50, 49-50
}

Sri Lanka has a policy of free healthcare delivered by a well-established, robust health system which includes both preventive and curative services. Our preventive health system provides a comprehensive package of services covering maternal and child care, immunization, disease surveillance and promotion of many preventive strategies. Sri Lanka has achieved many public health milestones; low maternal and infant mortality rates, high success, nearly $100 \%$ coverage, in childhood vaccination programs, bringing indigenous malaria cases to zero in 2012 and elimination of lymphatic filariasis in 2016. These milestones were achieved at a relatively low cost compared to other countries in the region ${ }^{1}$.

Curative health services are provided through a network of institutions structured in a three-tiered model; primary, secondary and tertiary care. In 2017, health expenditure as a share of GDP for Sri Lanka was 3.8\% and total per capita health expenditure was 159 USDs $^{2}$. There have also been major achievements in curative health care sector such as organ transplantation and bone marrow transplantation. However, due to the epidemiological and demographic transitions taking place over the last few decades, the curative system is now challenged with a new set of issues to deal with. Prevalence of non-communicable diseases (NCDs) which include hypertension, diabetes, ischaemic heart disease and stroke, has risen. NCDs were estimated to account for $83 \%$ of all deaths in Sri Lanka. Out of these $34 \%$ are due to cardiovascular diseases (CVD), 14\% cancers and 8\% due to chronic respiratory diseases ${ }^{3}$. The unavailability and lack of access to the preventive strategies and modern treatment could be accounted for the worst outcomes from the CVDs. Physicians are conscious of the fact that most of the patients with acute myocardial infarction are managed in medical wards due to the limited access to coronary care and cardiac procedural facilities. Although initiative in a few centres for thrombolysis in acute stroke care is commendable, there has not been an overall significant improvement in acute care, rehabilitation and prevention of stroke during the past decade. Cancer incidence has nearly doubled in twenty-five years and the pattern of cancer is also changing in Sri Lanka. Although there is free access to the cancer care services in the public sector, there are many unmet needs such as inadequate facilities for radiation therapy, lack of formal palliative care services, and a very limited capacity for research ${ }^{4}$.

Prevalence of chronic kidney disease (CKD) is on the rise and poses many challenges to our curative health care system. Diabetes, hypertension and CKD of unknown aetiology (CKDu) are the main contributors to the disease burden. Management of end stage kidney disease is too expensive and the available resources are unable to meet the burden of long-term treatment. Further, CKDu affects most vulnerable populations in rural areas and as a result provision of renal care facilities has become a major challenge.

Sri Lanka is rated as having the fastest growing aging population in the region. The elderly patient poses many challenges to the physician. Clinical presentation in elderly individuals can be very nonspecific, they may have several chronic diseases, taking multiple medications and likely to have complex social issues. An interdisciplinary/multidisciplinary service is therefore an absolute necessity to manage these complex physical, social and psychological needs. There is evidence to support the use of multidimensional packages, including pharmacological and psychosocial interventions; collaboration between different members of the primary-care team; involvement of patients and families and clear referral pathways; and appropriate supervision ${ }^{5}$. In Sri Lanka, there is no initiative yet to establish dedicated elderlycare units with specialist staff, owing to lack of human resources, infrastructure and funding. More physicians getting trained in geriatric medicine is encouraging.

Overcrowding of our tertiary care hospitals is a serious concern as it has a major impact on the quality of patient care. This has become a major concern as seen during frequent dengue epidemics. Although the institutions at different care levels are supposed to be streamlined, there is a lack of proper referral system between the institutions. As a result, patients often bypass the nearest primary care hospitals to attend secondary and tertiary care hospitals leading to overcrowding in these hospitals. According to the Annual Health Statistics of the Ministry of Health 2016,

This is an open-access article distributed under the terms of the Creative Commons Attribution License, which permits unrestricted use, distribution, and reproduction in any medium, provided the original author and source are credited. 
Bed Occupancy Rate (BOR) is variable; in general, secondary and tertiary care hospitals show high BOR, while District Hospitals record extremely low BOR ${ }^{3}$.

In addition to overcrowding, lack of diagnostic equipment and the most junior doctors getting overworked thus reducing their efficiency ${ }^{6}$, are other problems encountered in the curative care setting. Investigations such as $\mathrm{HbA} 1 \mathrm{C}$ are not available even in tertiary care hospitals. Most District Hospitals now accommodate Consultant Physicians, but there is lack of equipment and junior staff to provide a proper service to the patients. Further, there is a rising expectation among people for a better quality of health care. There is an increasing trend for people from high- and middleincome groups moving towards private health care expecting a better quality of care. This relieves the burden on free health care services to some extent.

Thus, the Sri Lankan healthcare system has to face the major challenge in combating noncommunicable diseases and chronic diseases. Currently, the system is not fully geared to provide these unmet needs. Effective interventions should involve strengthening the primary level curative care institutions ${ }^{7}$, an effective referral system to avoid overcrowding of secondary and tertiary care institutions ${ }^{7}$ and spending more on improvement on diagnostic and modern treatment facilities and extending these facilities to most vulnerable groups. However, given the current economic situation in the country, the extra funding needed for this purpose may not be available in the foreseeable future.

\section{Kamani Wanigasuriya}

Co-Editor, JCCP

E-mail:kamani303@gmail.com

\section{References}

1. Public health success in Sri Lanka WHO. http:// origin.searo.who.int/srilanka/documents/policy briefsfinal.pdf

2. World Health Organization Global Health Expenditure database. https://data.worldbank.org/indicator/SH.XPD. CHEX.GD.ZS?locations=LK

3. Department of Census and Statistics and Ministry of Health, Nutrition and Indigenous Medicine 2017. Sri Lanka Demographic and Health Survey 2016 Sri Lanka. http:// www.statistics.gov.Ik/social/DHS_2016a/FIST\% 2OPAGE_\&_CONTENTS.pdf

4. Gunasekara S, Seneviratne SA, Wijeratne T, Boot CM. Delivery of Cancer Care in Sri Lanka. Journal of Cancer Policy 2018; 18: 20-4. https://doi.org/10.1016/j.jcpo. 2018.10.001

5. Samaraweera D, Maduwage S. Meeting the current and future health-care needs of Sri Lanka's ageing population. South-East Asia Journal of Public Health, WHO, Regional Office for South-East Asia 2016; 05(2): 96-101. https:// www.ncbi.nlm.nih.gov/pubmed/28607235

6. Marasinghe MGCP, Abeyaratne D, Fonseka L, Gunatilake $\mathrm{SB}$. Work related fatigue and sleepiness at work among intern house officers of four hospitals in Sri Lanka. Journal of the Ceylon College of Physicians 2019; 50: 26-33.

7. Essential health services package 2019. Ministry of Health, Nutrition and Indigenous Medicine Sri Lanka. http:// www.health.gov.Ik/moh_final/english/public/elfinder/files/ publications/2019/SLESP-2019.pdf 